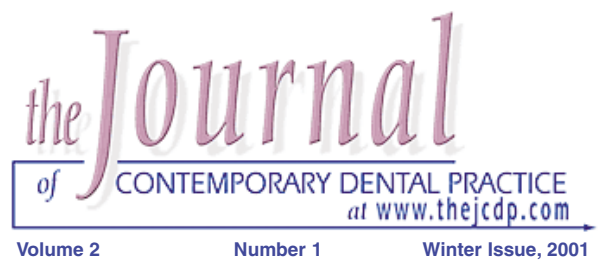

\title{
Clinical Dentin Hypersensitivity: Understanding the Causes and Prescribing a Treatment
}

\author{
Peter L. Jacobsen, PhD, DDS Professor, Dept of Pathology and Medicine;
} Gretchen Bruce, DDS, MBA Assistant Professor, Dept of Periodontology

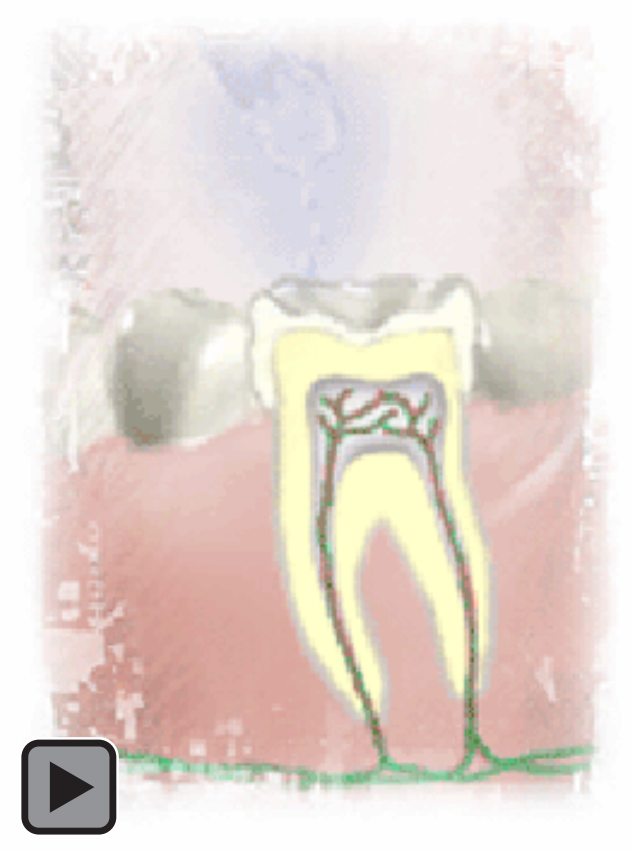

\begin{abstract}
Dentin hypersensitivity is a common condition of transient tooth pain associated with a variety of exogenous stimuli. There is substantial variation in the response to such stimuli from one person to another . Except for sensitivity associated with tooth bleaching or other tooth pathology, the clinical cause of dentin hypersensitivity is exposed dentinal tubules as a result of gingival recession and subsequent loss of cementum on root surfaces. The most widely accepted theory of how the pain occurs is Brännström's hydrodynamic theory of dentin hypersensitivity. Dentinal hypersensitivity must be differentiated from other conditions that may cause sensitive teeth prior to treatment. Three principal treatment strategies are used. Dentinal tubules can be covered by gingival grafts or dental restorations. The tubules can be plugged using compounds that can precipitate together into a large enough mass to occlude the tubules. The third strategy is to desensitize the nerve tissue within the tubules using potassium nitrate. Several over-the-counter products are available to patients to treat this condition.

Keywords: Dentin hypersensitivity, Brännström's theory, dentin tubules, hypersensitive teeth
\end{abstract}

@ Seer Publishing 


\section{Introduction}

Dentin hypersensitivity is a common condition of transient tooth pain caused by a variety of exogenous stimuli. The condition affects more than 40 million people in the United States annually. ${ }^{1} \quad$ The exogenous stimuli include thermal (cold), tactile (touch), or osmotic changes (sweets or drying the surface). ${ }^{2}$ While extreme stimuli can make all teeth hurt, the "hyper" sensitivity means painful response to stimuli not normally associated with pain. The response to a stimulus varies from person to person due to differences in pain tolerance, environmental factors, and emotional state. ${ }^{3}$

Although most people can tolerate a wide range of oral changes, some patients experience discomfort to the extent that it interferes with their eating patterns and oral hygiene habits. For these hypersensitive patients, it is appropriate to evaluate the cause and to prescribe a treatment.
The primary underlying clinical cause for dentin hypersensitivity is exposed dentinal tubules. This clinical condition allows for fluid flow within the tubules (the hydrodynamic theory), creating pain. The only hypersensitivity not associated with this etiology is the transient spontaneously resolving hypersensitivity associated with the dental bleaching process.

The most common clinical cause for exposed dentinal tubules is gingival recession. ${ }^{4}$ The recession may or may not be associated with bone loss. If bone loss occurs, then more dentinal tubules can be exposed. A wide range of mechanisms has been proposed as causes of recession. (See Table 1) When gingival recession occurs, cementum is exposed. Cementum, being a very thin outer protective layer on dentin, is easily abraded or eroded away. This leaves the underlying dentin, which consists of tubules which contain the protoplasmic projections (little arms) of the cells (odontoblasts) within the tooth pulp chamber. These cells contain nerve endings and when disturbed, depolarize (fire off). ${ }^{5,6}$ This neural discharge is interpreted as pain. The pain has a rapid onset and is usually of short duration, but it can persist as a dull ache. ${ }^{2}$

As noted earlier, the most accepted theory of how the pain occurs is Brännström's hydrodynamic theory of dentin hypersensitivity. 7,8 This postulates that fluids within the tubule are disturbed either by temperature changes or physical osmotic changes and that these fluid changes (movements) stimulate a baroreceptor (a nerve receptor sensitive to pressure) which leads to

\section{Table 1. Common Reasons for Gingival Recession}

1. Inadequate attached gingiva

2. Prominent roots

3. Toothbrush abrasion

4. Pocket reduction periodontal surgery

5. Oral habits resulting in gingival laceration, i.e., traumatic tooth picking eating hard foods

6. Excessive tooth cleaning

7. Excessive flossing

8. Gingival loss secondary to specific diseases, i.e., NUG, periodontitis, herpetic gingivostomatitis

9. Crown preparation 
neural discharge (depolarization). A scientific hypothesis for dentinal hypersensitivity is informative, but exposed dentinal tubules is the clinical reality that leads to the hypersensitivity.

Once the dentinal tubules are exposed, there are often oral processes or habits that keep them exposed. These include poor plaque control, enamel wear, cervical erosion, incorrect oral

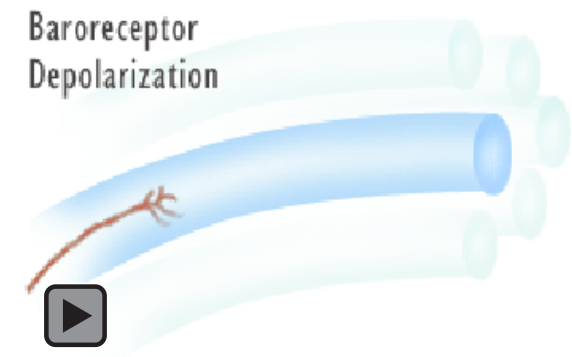

hygiene technique, and exposure to acids. (See Table 2) Many of these reasons are the same as those that caused the gingival recession in the first place. Since dentinal tubules naturally sclerose and plug themselves up in the oral environment, over time, treatment should focus on eliminating factors associated with continued dentinal tubule exposure.

\section{Treatment Strategies}

Treatment of dentin hypersensitivity is challenging for both the patient and the healthcare provider for two reasons. It is difficult measuring/comparing different patient's pain and it is difficult for patients to change the habits that initially caused the problem. Hypersensitivity can resolve without treatment or may require several weeks of desensitizing agents before improvement is seen. ${ }^{9}$ There are two principal treatment options. You can:

- plug the dentinal tubules preventing fluid flow, or

- desensitize the nerve, making it less responsive to stimulation. (See Table 3)
All of the current treatment modalities address these two options.

To date, no single agent or form of treatment has been found effective for all patients. Solutions, gels and pastes-containing fluorides in varying compounds and percentages, calcium hydroxide, strontium chloride, potassium nitrate, sodium citrate, formaldehyde, or potassium or ferric oxalate have all been tried, with varying degrees of success. $^{2}$

\section{Nerve Desensitization}

Currently there is only one compound that claims to desensitize the nerve. That compound is potassium nitrate. To get the ADA Seal of Acceptance for treating dentin hypersensitivity, at least two double-blind clinical studies must be completed demonstrating a statistically significant sensitivity reduction. ${ }^{10}$ All over-the-counter desensitization dental products with ADA and FDA approval currently contain potassium nitrate. It is theorized that potassium nitrate penetrates through the dentinal tubules to the nerve. The potassium ion may depolarize the nerve and prevent it from repolarizing, thereby, preventing it from sending pain signals to the brain. Currently the ADA accepted desensitizing toothpastes are Crest $^{\circledR}$ Sensitivity Protection Fluoride Toothpaste, Orajel ${ }^{\circledR}$ Sensitive Pain Relieving Toothpaste for Adults, Colgate ${ }^{\circledR}$; Sensitive Maximum Strength Toothpaste, and Protect ${ }^{\circledR}$; Sensitive Teeth Gel Toothpaste. Currently accepted ADA products can be reviewed on the Internet by going to www.ada.org and looking under ADA Seal of Acceptance in the public information section. It is possible in the future that desensitizing toothpaste may contain two active ingredients. One would be designed to penetrate the tubule and desensitize the nerve and the other to occlude the tubule.

Table 2. Reasons for Continued Dentinal Tubular Exposure

1. Poor plaque control, i.e., acidic bacterial byproducts

2. Excess oral acids, i.e., sodas, fruit juice, swimming pool chlorine, bulimia

3. Cervical decay

4. Toothbrush abrasion

5. Tartar control toothpaste 


\section{Covering or Occluding Dentinal Tubules}

Prior to the development of potassium nitrate as an OTC desensitizer, occluding or sclerosing the open dentinal tubules was the primary method used to control hypersensitivity. Now that approach, along with covering the exposed dentinal tubules, is primarily reserved as a professional treatment method. Methods to physically cover the dentinal tubules include periodontal surgery to bring the tissue over the surface of the exposed dentinal tubules or placing a dental restoration over them, protecting the tubules from the oral environment. The other means of covering the dentinal tubules is to seal the surface either with a dentinal sealer or a composite/glass ionomer restoration.

The alternative approach to block the tubules is to occlude/sclerose (plug) the inside of the dentinal tubules. Tubule occlusion can occur either natu- rally over time or through professional intervention. One natural occluder is calcium precipitation, i.e., calculus (tartar) on the surface of the tooth. Prevention of calculus formation by using tartar control toothpaste, though an advantage in minimizing gingivitis and enhancing cosmetic appearance, may prolong dentin hypersensitivity after dental cleaning in some patients. Another way that nature has of minimizing or eliminating hypersensitivity is to sclerose the dentinal tubules from within. As patients age, they tend to have less sensitivity because the tubules are naturally sclerosed with secondary dentin.

Professional (by prescription or office applied) dental products, which occlude the dentinal tubules, comprise the vast majority of products available to control hypersensitivity. One subgroup of these products are those which contain ions or salts which are hypothesized to precipitate

Table 3. Treatment Options

1. Desensitize the nerve

a. potassium nitrate

2. Cover the dentinal tubules

a. periodontal surgery/grafting

b. composite/glass ionomer restoration

c. crown placement

d. plug (sclerose) the dentinal tubules

1. ions/salts

a. stannous fluoride

b. sodium fluoride/stannous fluoride combination

c. potassium oxalate

d. ferrous oxide

e. strontium chloride

f. in combination with an adhesive

2. precipitates - proteins/amino acids

a. glutaraldehyde

3. resins

a. dentin sealers

b. methyl methacrylate 

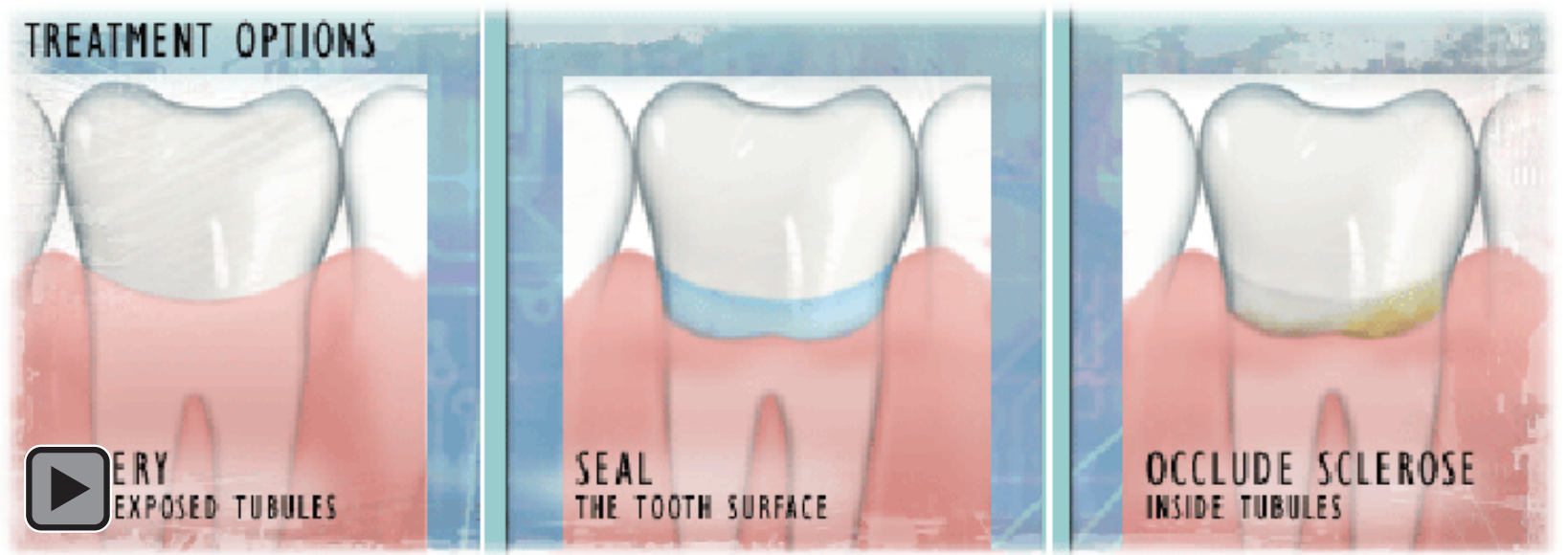

within the tubule. This includes the stannous ion in stannous fluoride, (Gel Kam ${ }^{\circledR}$ Pro-Dentx ${ }^{\circledR}$, and OMNI product lines of stannous fluoride). Clinical studies have documented reduction in dentinal hypersensitivity using a solution that contained stannous, sodium, and hydrogen fluoride (Dentin Bloc). Symptoms were lessened with regular usage. ${ }^{11,12}$ The stannous ions may precipitate together into a large enough mass to occlude dentinal tubules. Though these occluding technologies were sometimes successful, none of them currently are used in OTC toothpastes, which have received the ADA Seal of Acceptance for dentin hypersensitivity. Oxalate is another ion used in professionally applied desensitizing solutions (Protect Dentin Block and Sensodyne Sealant). The oxalate ions react with the calcium in the tooth to form insoluble calcium oxalate crystals that occlude the tubules. ${ }^{13,14}$

Strontium ions also have the property of precipitating together forming a mass that occludes the tubules. Strontium chloride was used previously in an OTC toothpaste preparation, however, this preparation is no longer available. Other products designed to occlude the tubules are those such as Gluma which contains glutaraldehyde which is hypothesized to precipitate and coagulate proteins/amino acids within the tubule. Gluma also contains a methyl methacrylate to seal the tubules. Other bonding/sealing agents containing methyl methacrylate or some type of surface precipitant that have been recommended for chairside treatment of dentinal sensitivity are All Bond DS Desensitizer, Micro prime, and ConfiDental. However, there are currently no clinical studies published that support in vivo experience for these products.

\section{Treatment of Dentin Hypersensitivity}

When a patient presents with what appears to be sensitive dentin, the initial diagnosis should eliminate any possible reasons such as decay, cracked tooth, or irreversible pulpitis that may mimic dentin hypersensitivity. The next appropriate step, once the problem has been identified as dentin hypersensitivity, is to identify the reason for the exposed dentinal tubules and to see if the etiology/process causing the hypersensitivity can be eliminated.

If the hypersensitivity persists after the etiology is removed, then the simplest and most cost ef fective approach is to place the patient on an OTC desensitizing toothpaste. This can be done by following manufacturer instructions which is usually to brush the teeth with the toothpaste twice a day for at least two weeks. Depending on the response and the severity of the problem, some patients and dentists have elected to place the desensitizing toothpaste in a "fluoride tray" or zero clearance-bleaching tray for five minutes twice a day, increasing the duration and the intensity of the application. If the OTC product is not effective, then an in-office treatment with one of the ionic salts or a protein precipitant would be the next appropriate step.

\section{Treatment of Dentin Hypersensitivity} Depending on the patient's response to these treatments and depending on the etiology and severity of the problem, the next step would be to apply dentin bonding/sealer to the surface or restore the affected teeth with composite restorations covering the exposed dentinal tubule area. 
When the patient first presents with dentin hypersensitivity, they should be informed of the series of steps that may be necessary to eliminate the problem. If they are informed at the beginning relative to all the possible steps including the possible need for restorations, then they are less frustrated and less suspicious as each new step fails and the next step that is suggested is increasingly expensive. Also, when fully informed, the patient is put in charge of the decision making process. They decide when they want to go to the next step, since they know what the next step will be. In rare occasions no matter what procedure is tried, the hypersensitivity may persist.

The patient should also be informed at the beginning that the ultimate way of desensitizing a tooth is extirpation of the dental pulp; endodontics. This will clearly desensitize the tooth, but requires additional restoration for its final treatment. If the patient is informed about this final solution in the beginning, then they have the full treatment plan and fully understand their and your choices relative to this annoying problem. (See Table 4).

Dental bleaching has been reported to cause a number of side effects, including tooth sensitivity, gingival irritation, tooth pain, tingling of the tissues, and sore throat. Tooth sensitivity and gingival irritation are the most frequently reported complaints. The hypersensitivity that occurs in association with bleaching has been attributed to patient factors, length of exposure to the bleaching agent, the concentration of the bleaching agent, $\mathrm{pH}$ of the whitening solution, and tray factors. Patients, especially those who already have exposed dentin or already have some type of hypersensitivity or those with larger pulps, should be warned that they may have a greater risk of hypersensitivity secondary to bleaching. Since plugging the tubules is not an appropriate option, because the bleach is intended to penetrate into the tubules to decolorize (oxidize) the nonfunctional staining proteins and materials, the only reasonable treatment is to desensitize the nerve with potassium nitrate.

If a patient has sensitivity secondary to bleach use, they should be instructed to reduce the frequency or duration or discontinue the bleaching process, depending on the severity of the sensitivity reaction. Another option is to recommend a bleach product that contains potassium nitrate, or recommend the patient desensitize their teeth with a potassium nitrate containing toothpaste for several days prior to using the bleach. This could entail using bleaching trays to hold the potassium nitrate containing toothpaste in place around the teeth. This would desensitize the nerves and allow the patient to use the bleach.

The higher the concentration of bleach, the greater the risk of sensitivity. Ten percent carbamide peroxide causes less sensitivity, but the bleaching process is slow, though it will eventually get to the same level of whiteness as the higher concentrations. ${ }^{15}$ Twenty percent and higher carbamide peroxide accelerates the bleaching process, but sensitivity may be intolerable.

Based on that, for optimum bleaching time and minimum sensitivity a fifteen percent bleach containing potassium nitrate might be an ideal combination. A plain fifteen percent bleach used after the teeth are pretreated with potassium nitrate would also optimize bleaching and minimize sensitivity.

Table 4. Treatment Steps

1. Thorough exam to identify etiology and eliminate tooth fracture and irreversible pulpitis.

2. Potassium nitrate containing product/toothpaste $2 \mathrm{x}$ day for at least 2 weeks.

3. Potassium nitrate containing product in a tight fitting dental tray.

4. In-office tubule occluding product.

5. In-office tubule sealer.

6. Dental restoration, or a periodontal surgical procedure, that covers the exposed dentin.

7. Endodontic procedure to remove the pulp. 


\section{Conclusion}

Dentin hypersensitivity is a problem that plagues many patients. The initial or continued clinical cause, in the majority of situations, is gingival recession. Recession exposes root surfaces and once the thin cemental layer is removed, the dentinal tubules are exposed. Once the tubules are exposed, the protoplasmic projections of the odontoblasts are exposed to the environment. Any change in fluid pressure within the tubule is detected by the odontoblast. The nerve then depolarizes and the patient has pain.

The initial treatment choices to treat dentin hypersensitivity are to cover up or occlude the tubules or to desensitize the nerves so they are not as responsive to stimulation. The only product available to desensitize the nerve is potassium nitrate. This compound is the active ingredient in all OTC desensitizing toothpastes. Occluding or covering up the tubules can be accomplished using many products. Some of these seal the outer edge of the tubules and others precipitate salts or proteins within the tubules. The end result is the tubules are blocked; preventing fluid movement within them.

Patients should be informed of all the possible steps that may be necessary to eliminate their hypersensitivity. The first step should be to identify and eliminate the cause. The next is to rectify the recession or seal the exposed dentinal tubules from the oral environment. If the patient is thoroughly informed of all the possible steps then they become a partner in the treatment process, identifying for the dentist when they want to proceed to the next step. This minimizes frustration on the patient's part and facilitates the process by which the dentist solves the problem.

\section{References}

1. Kanapka JA. Current treatment for dentinal hypersensitivity. A new agent. Compend Contin Educ Dent 1982;(Suppl 3 ):S118-20. No abstract available.

2. Addy M, Mostafa P, Newcombe RG. Effect of plaque of five toothpastes used in the treatment of dentin hypersensitivity. Clin Prev Dent 1990;12: 28-33.

3. Orchardson R, Collins WJ. Clinical features of hypersensitive teeth. Br Dent J 1987;162: 253-6. No abstract available.

4. Bal J, Kundalgurki S. Anbsp;Tooth sensitivity prevention and treatment. Oral Health 1999;89:33-4, 37-8, 41. Review. No abstract available.

5. Brannstrom M, Astrom A. The hydrodynamics of the dentine; its possible relationship to dentinal pain. Int Dent J 1972;22: 219-27. No abstract available.

6. Berman LH. Dentinal sensation and hypersensitivity. A review of mechanisms and treatment alternatives. J Periodontol 1985;56: 216-22. Review.

7. Brannstrom M. The hydrodynamic theory of dentinal pain: sensation in preparations, caries, and the dentinal crack syndrome. J Endod 1986;12: 453 No abstract available.

8. Brannstrom M, Johnson G, Nordenvall KJ. Transmission and control of dentinal pain: resin impregnation for the desensitization of dentin. J Am Dent Assoc 1979;99: 612-8.

9. Carranza FA, Newman MG. Clinical Periodontology, ed. 8, Philadelphia, 1996, W.B. Saunders Company.

10. American Dental Association Council on Scientific Affairs: Acceptance Program Guidelines Products for the Treatment of Dentinal Hypersensitivity, May 1998.

11. Thrash WJ, Jones DL, Dodds WJ. Effect of a fluoride solution on dentinal hypersensitivity. Am J Dent 1992;5: 299-302.

12. Blong MA, Volding B, Thrash WJ, Jones DL. Effects of a gel containing 0.4 percent stannous fluoride on dentinal hypersensitivity. Dent Hyg (Chic) 1985;59: 489-92. No abstract available.

13. Greenhill JD, Pashley DH. The effects of desensitizing agents on the hydraulic conductance of human dentin in vitro. J Dent Res 1981;60: 686-98.

14. Wichgers TG, Emert RL. Dentin hypersensitivity. Gen Dent 1996;44: 225-30; quiz 231-2. Review . No abstract available.

15. Mokhlis GR, Matis BA, Cochran MA, Eckert GJ. A clinical evaluation of carbamide peroxide and hydrogen peroxide whitening agents during daytime use. J Am Dent Assoc 2000;131: 1269-77. 
Peter L. Jacobsen, PhD, DDS

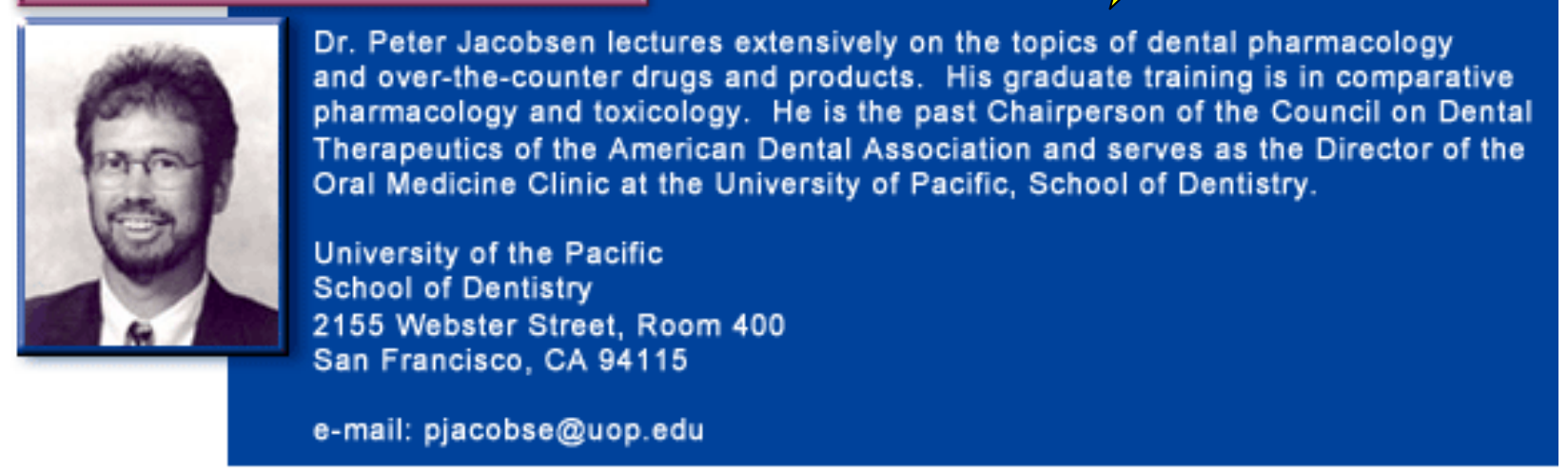

\section{Gretchen J. Bruce, DDS, MBA}

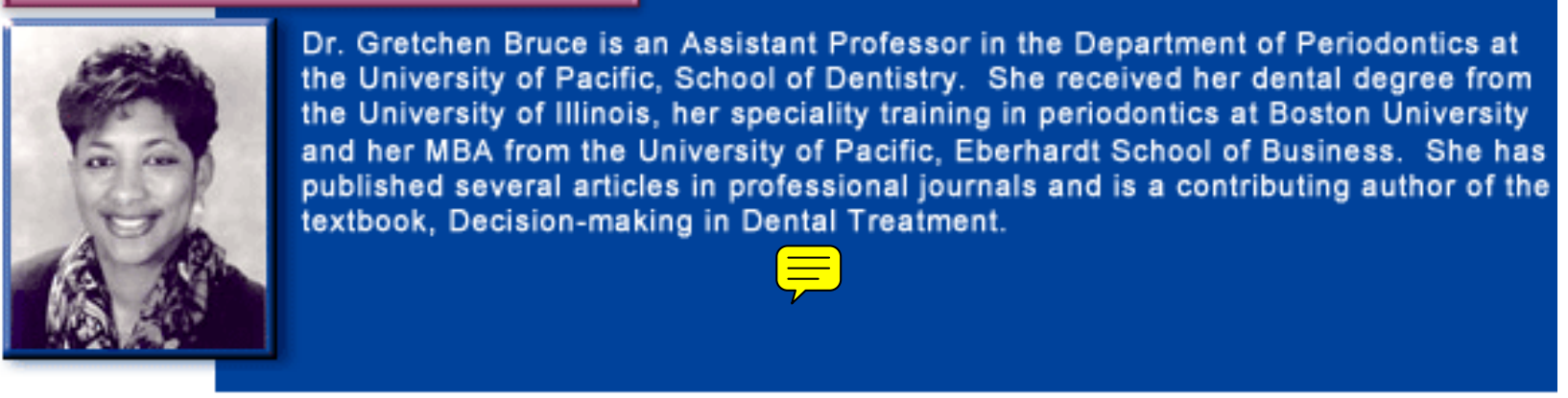

